\title{
Differential Effects of Unsaturated Fatty Acids and Saturated Fatty Acids on Lipotoxicity and Neutral Lipid Accumulation in Neuro-2a Cells
}

\author{
CJ Urso and Heping Zhou* \\ Department of Biological Sciences, Seton Hall University, South Orange, NJ 07079, USA
}

*Corresponding author: Heping Zhou, Department of Biological Sciences, Seton Hall University, South Orange, NJ 07079, USA

\section{ARTICLE INFO}

Received: 幽 July 14, 2021

Published: 慧 July 27, 2021

Citation: CJ Urso, Heping Zhou. Differential Effects of Unsaturated Fatty Acids and Saturated Fatty Acids on Lipotoxicity and Neutral Lipid Accumulation in Neuro-2a Cells. Biomed J Sci \& Tech Res 37(3)-2021. BJSTR. MS.ID.006017.

Abbreviations: ALA: $\alpha$-Linolenic Acid; ARA: Arachidonic Acid; BMI: Body Mass Index; DAG: Diacylglycerol; DHA: Docosahexaenoic Acid; ER: Endoplasmic Reticulum; FFA: Free Fatty Acids; LD: Lipid Droplet; LA: Linoleic Acid; N2a: Neuro-2a; OA: Oleic Acid; PA: Palmitic Acid; SFA: Saturated Fatty Acids; TAG: Triacylglycerol; UFA: Unsaturated Fatty Acids; BODIPYTM 493/503, 4,4-Difluoro-1,3,5,7,8-Pentamethyl-4-Bora-3a,4a-Diaza-s-Indacene; BSA: Bovine Serum Albumin; DAPI: 4',6-diamidino-2-phenylindole

\begin{abstract}
Long-chain free fatty acids (FFA) play many important roles in cell growth and metabolism. Accumulation of excess saturated fatty acids (SFA) leads to deleterious lipotoxic effects in non-adipose tissues while unsaturated fatty acids (UFA) often exert protective effects against SFA lipotoxicity, yet the lipotoxic effects of SFA in neuronal cells have not been well characterized. This study examined the differential effects of SFA and UFA on the viability of Neuro-2a (N2a) cells and the accumulation of neutral lipids in these cells. Our study found that all the UFA tested, namely oleic acid (OA), linoleic acid (LA), $\alpha$-linolenic acid (ALA), and docosahexaenoic acid (DHA), were able to abolish PAinduced decrease in cell viability regardless of the position of the double bond or degree of unsaturation, and that $200 \mu \mathrm{M} \mathrm{LA}, \mathrm{OA}$, and DHA significantly enhanced the amount of neutral lipid staining than BSA control while PA did not, suggesting that LA, OA, and DHA, but not PA, increased the amount of neutral lipid synthesis and accumulation. The neutral lipid staining also appeared more in particulates in UFA-treated cells than PAtreated cells, suggesting that UFA, but not PA, enhanced LD formation. We also found that the amount of neutral lipid staining in cells co-treated with UFA and PA was comparable to that in cells treated with BSA or PA alone, and that the neutral lipid staining in cells co-treated with UFA and PA appeared more concentrated in particulates than PA-treated cells, suggesting that UFA may not enhance neutral lipid accumulation, but may increase LD formation in PA-treated cells. Our results suggest that UFA and SFA have differential effects on cell viability, neutral lipid accumulation, and LD formation in N2a cells. Further studies will be needed to examine the role of LD formation in UFA protection against PA lipotoxicity.
\end{abstract}

Keywords: Lipid Droplets; Lipoprotection; Unsaturated Fatty Acids; Palmitic Acid; Lipotoxicity

\section{Introduction}

Long-chain free fatty acids (FFA) play many important roles in cell growth and metabolism, as structural components of biological membranes and as mediators of cell signaling. Common longchain FFA found in human plasma include saturated fatty acids (SFA), such as palmitic acid (PA; 16:0) and stearic acid (18:0), and unsaturated fatty acids (UFA) which may vary in the position of the double bond and in the degree of unsaturation, including oleic acid
(OA; 18:1n-9), linoleic acid (LA; 18:2n-6), $\alpha$-linolenic acid (ALA; 18:3n-3), and docosahexaenoic acid (DHA; 22:6n-3) [1]. After entry into cells, fatty acyl CoA synthetase catalyzes the conversion of fatty acids into fatty acyl-CoA, which may then be catabolized to generate energy or anabolized to produce a range of molecules including second messengers, hormones, and diacylglycerols (DAG). DAG may enter the phospholipid synthesis pathways or be converted 
to triacylglycerols (TAG), which may be subsequently incorporated into lipid droplets (LD) [2].

One of the hallmarks of metabolic diseases is the accumulation of excess fatty acids in non-adipose tissues [3], which may lead to deleterious lipotoxic effects including cellular dysfunction and death [4]. PA has been reported to induce lipotoxicity in many cell types, including cardiomyocytes [5], hepatocytes [6], and neural stem cells $[7,8]$. In contrast, $\omega-3$ fatty acids enhance hippocampal neurogenesis and promote synaptic plasticity [9]. OA ameliorates PA-induced endoplasmic reticulum (ER) stress in exocrine pancreas cells [10] and HepG2 cells [11]. LA also protects cultured mouse embryonic cortical neurons from glutamate-induced excitotoxicity [12]. There is evidence suggesting that UFA-induced esterification and sequestration of PA into neutral lipids and LD may contribute to their lipoprotective effects. LD, consisting of a neutral lipid core containing TAG, sterol esters, and fatty acids surrounded by a monolayer of phospholipids embedded with numerous proteins, is found in many types of cells. LD not only acts as lipid storage sites, but also participates in membrane biogenesis, hormone synthesis, intracellular signaling, protein storage, and protein degradation $[13,14]$.

LD biogenesis and degradation has been suggested to play important roles in buffering the intracellular levels of toxic lipid species [14]. For example, arachidonic acid (ARA; C20:4n-6)induced LD formation is associated with its protection against PA-induced lipotoxicity in C2C12 myocytes [15]. OA-induced TAG synthesis and LD formation is associated with its protection against PA-induced lipotoxicity in primary syncytiotrophoblasts [16], CHO cells [17], and $\beta$-cells [18]. Fatty acid accumulation is reportedly enhanced in the brains of metabolic syndrome patients [19]. While neurons are not energy-storing cells and their use of fatty acids as an energy source is minimal, they do contain LD [20]. High fat diet causes obesity and loss of myenteric neurons in Swiss mice [21]. High fat diet also affects the hypothalamic proteome indicative of cellular stress, altered synaptic plasticity and mitochondrial function in mice [22]. This study was therefore designed to examine how SFA and UFA differentially affect cell viability and neutral lipid accumulation in murine neuroblastoma Neuro-2a (N2a) cells.

\section{Materials and Methods}

\section{Reagents}

LA, OA, PA, ALA, DHA, and fatty acid-free bovine serum albumin (BSA) were purchased from Sigma-Aldrich (St. Louis, MO, USA). To prepare BSA-conjugated fatty acids, fatty acids were dissolved in $100 \%$ ethanol at $400 \mathrm{mM}$. FAs were then conjugated with BSA in
PBS at 2.5:1 molar ratio to make $5 \mathrm{mM}$ FA solutions [23]. Conjugated fatty acids were then filtered, aliquoted, and stored at $-80^{\circ} \mathrm{C}$.

\section{Cell Culture}

Murine Neuro-2a (N2a) neuroblastoma cells were purchased from American Type Culture Collection (Manassas, VA, USA). These cells were cultured in Eagle's minimum essential media (Lonza; Walkersville, MD, USA) supplemented with $10 \%$ (v/v) fetal bovine serum (Invitrogen; Carlsbad, CA, USA) and 1\% (v/v) penicillinstreptomycin (Sigma-Aldrich) and maintained in a humidified environment with $5 \% \mathrm{CO} 2$ at $37^{\circ} \mathrm{C}$. All experiments were conducted with cells passaged fewer than 20 times.

\section{MTT Assay}

Cell viability was determined based on the ability of cellular nicotinamide adenine dinucleotide phosphate (NADPH)dependent oxidoreductase enzymes to reduce the tetrazolium dye, 3-(4,5-dimethyl-2-thiazolyl)-2,5-diphenyl-2H-tetrazolium bromide (MTT; Sigma-Aldrich), to insoluble formazan. Cells were seeded in 96-well plates at $1.0 \times 10^{4}$ cells/well. After overnight incubation, cells were administered with different treatments in serum free media with BSA as the negative control. At $2 \mathrm{~h}$ prior to the completion of treatment, MTT reagent was added to the wells at a final concentration of $0.5 \mathrm{mg} / \mathrm{ml}$ and incubated for another $2 \mathrm{~h}$. At the end of incubation, the formazan precipitates were solubilized in isopropanol with $0.04 \mathrm{M} \mathrm{HCl}$, and the absorbance was read at 570 $\mathrm{nm}$ and $650 \mathrm{~nm}$ for reference absorbance to correct for nonspecific background absorption on the VarioSkan Lux microplate reader (Thermo; Waltham, MA, USA).

\section{Annexin V Assay}

Cells were seeded in 12-well plates at $1.0 \times 10^{5}$ cells/well. After overnight incubation, cells were treated for $24 \mathrm{~h}$ with different concentrations of PA or BSA as the negative control in serum free media. At the end of treatment, cells were collected by trypsinization, spun down, and then washed with binding buffer (140 mM NaCl, 4 mM KCl, 0.75 mM MgCl2, 10 mM HEPES, 2.5 mM $\mathrm{CaCl} 2$ ) before being resuspended in staining solution containing 5\% Annexin V-FITC (BD Biosciences; San Jose, CA, USA) and $2 \mu \mathrm{g} /$ $\mathrm{mL}$ propidium iodide (PI). Following incubation at RT for $15 \mathrm{~m}$ in the dark, cells were analyzed on the MACSQuant Analyzer 10 flow cytometer (Miltenyi Biotec; Auburn, CA, USA). The fluorescent intensities of FITC and PI were quantified. The central population of forward scatter (FSC) vs. side scatter (SSC) flow cytometry events was inclusively gated to omit debris. Singlets were then sub-gated by FSC-A vs. FSC-H. Flow cytometry data were then analyzed using FlowJo10 (BD Biosciences; Ashland, OR, USA). 


\section{Terminal deoxynucleotidyl transferase dUTP nick end labeling (TUNEL) Assay}

TUNEL assay was performed following manufacturer's instructions. Briefly, cells were seeded in chamber slides at $2.5 \mathrm{x}$ $10^{4}$ cells/chamber. After overnight incubation, cells were treated for $24 \mathrm{~h}$ with different concentrations of PA or BSA control in serum free media. After fixation with 4\% paraformaldehyde for $25 \mathrm{~m}$ at $4^{\circ} \mathrm{C}$, cells were washed, permeabilized with $0.2 \%$ Triton X-100 for $5 \mathrm{~m}$, washed, and then incubated with a reaction mix containing terminal deoxynucleotidyl transferase (TdT) and fluorescein-12dUTP (Roche; Indianapolis, IN, USA) for $90 \mathrm{~m}$ at $37^{\circ} \mathrm{C}$. After washing, cells were stained with PI, and then visualized under the FluoView FV1000 confocal microscope (Olympus; Center Valley, PA, USA).

\section{Neutral Lipid Quantitation by Flow Cytometry}

Cells were seeded in 24-well plates at a density of $0.5 \times 10^{5}$ cells/well. Following treatment, cells were incubated with $5 \mu \mathrm{M}$ 4,4-Difluoro-1,3,5,7,8-Pentamethyl-4-Bora-3a,4aDiaza-s-Indacene (BODIPY $^{\mathrm{TM}}$ 493/503; ThermoFisher; Waltham, MA, USA), a fluorescent marker for neutral lipids, in serum-free media for 30 $\mathrm{m}$ at $37^{\circ} \mathrm{C}$. Cells were then trypsinized, rinsed, and resuspended in ice-cold PBS, and analyzed by flow cytometry.

\section{Neutral Lipid Imaging}

For bright-field imaging of neutral lipids, cells were seeded on coverslips and treated as indicated. At the end of treatment, cells were fixed with $4 \%$ paraformaldehyde for $20 \mathrm{~m}$ at $4^{\circ} \mathrm{C}$, washed, equilibrated in $60 \%$ isopropanol for $5 \mathrm{~m}$, and incubated with $0.5 \%$ Oil Red 0 for $20 \mathrm{~m}$ at room temperature. Cells were then washed, mounted, and imaged under the Motic Panthera light microscope
(Schertz, TX, USA). For fluorescent imaging of neutral lipids, cells were incubated with $2 \mu$ M Nile Red or $5 \mu$ M BODIPYTM $493 / 503$ for $30 \mathrm{~m}$ following treatment. Cells were then rinsed with PBS and fixed with $4 \%$ paraformaldehyde for $20 \mathrm{~m}$ at $4{ }^{\circ} \mathrm{C}$. After rinsing with PBS, cells were stained with $1 \mu \mathrm{g} / \mathrm{ml}$ DAPI for $10 \mathrm{~m}$ in the dark, and then imaged under FluoView FV1000 confocal microscope (Olympus; Center Valley, PA, USA).

\section{Statistical Analysis}

Data were analyzed using GraphPad Prism 6 (Graphpad Software; San Diego, CA, USA) and presented as mean \pm SEM. Oneway or two-way ANOVA followed by Tukey's multiple comparison test was used to determine statistical significance. $p<0.05$ was considered statistically significant.

\section{Results}

\section{PA Treatment Reduced the Viability of N2a Cells}

To determine the effects of PA treatment on the viability of N2a cells, N2a cells were treated with different concentrations of PA ranging from $25 \mu \mathrm{M}$ to $300 \mu \mathrm{M}$ or BSA control in serum free media for $24 \mathrm{~h}$, and their viability was assessed by MTT assay. The viability of N2a cells was significantly decreased following $24 \mathrm{~h}$ treatment with $25 \mu \mathrm{M}$ PA as compared to the BSA control. As the concentration of PA was increased, the viability of N2a cells continued to decrease (Figure 1A). N2a cells were then treated with $200 \mu \mathrm{M}$ PA or BSA control for 6,24 , or $48 \mathrm{~h}$. At $6 \mathrm{~h}$ after PA treatment, no significant difference in cell viability was detected. However, $200 \mu \mathrm{M}$ PA significantly decreased the viability of N2a cells at $24 \mathrm{~h}$ and $48 \mathrm{~h}$ post-treatment (Figure 1B).
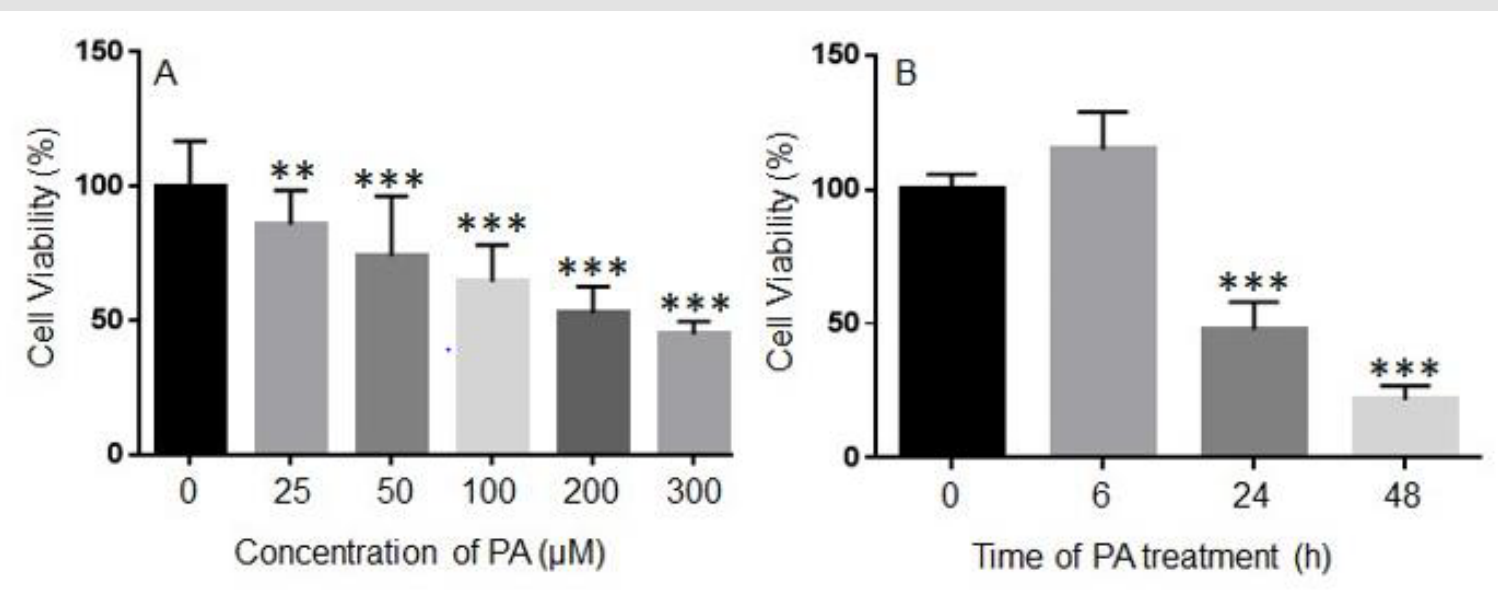

Figure 1: Concentration- (A) and time-dependent (B) effects of palmitic acid (PA) on the viability of N2a cells. N2a cells were treated with different concentrations of PA for $24 \mathrm{~h}$ (A) or with $200 \mu \mathrm{M}$ PA for different periods of time (B) in serum free media, and their viability was assessed by MTT assay. The values presented were representative of three independent experiments with triplicate measurements (mean \pm SEM). Statistical analysis was performed using one-way ANOVA followed by Tukey's multiple comparison test. ${ }^{* *} \mathrm{p}<0.01$ vs. control; ${ }^{* * *} \mathrm{p}<0.001$ vs. control. 


\section{PA Treatment Induced Cell Death in N2a cells}

To determine whether PA-induced decrease in N2a viability was associated with increased cell death, N2a cells were treated with different concentrations of PA ranging from $25 \mu \mathrm{M}$ to $200 \mu \mathrm{M}$ or BSA control in serum free media for $24 \mathrm{~h}$, stained with Annexin V-FITC and propidium iodide (PI), and analyzed by flow cytometry. The number of Annexin V-positive and PI-positive cells in BSAtreated control was minimal. Treatment with $25 \mu \mathrm{M}$ PA did not significantly enhance the number of Annexin V- or PI-positive cells. The number of Annexin V- and PI-positive cells began to increase following treatment with $50 \mu \mathrm{M}$ PA for $24 \mathrm{~h}$ and was significantly enhanced following treatment with $100 \mu \mathrm{M}$ PA for $24 \mathrm{~h}$. This trend continued to amplify in cells treated with $200 \mu \mathrm{M}$ PA (Figures 2A \& 2B). PA-induced cell death was also examined by terminal deoxynucleotidyl transferase dUTP nick end labeling (TUNEL) assay. There were hardly any cells stained positive by TUNEL assay in BSA-treated samples. Treatment with $25 \mu \mathrm{M}$ or $50 \mu \mathrm{M}$ PA for 24 $h$ did not significantly increase the number of TUNEL positive cells. The percentage of TUNEL positive cells was significantly increased following treatment with 100 or $200 \mu \mathrm{M}$ PA for 24 h (Figures 2C \& 2D).
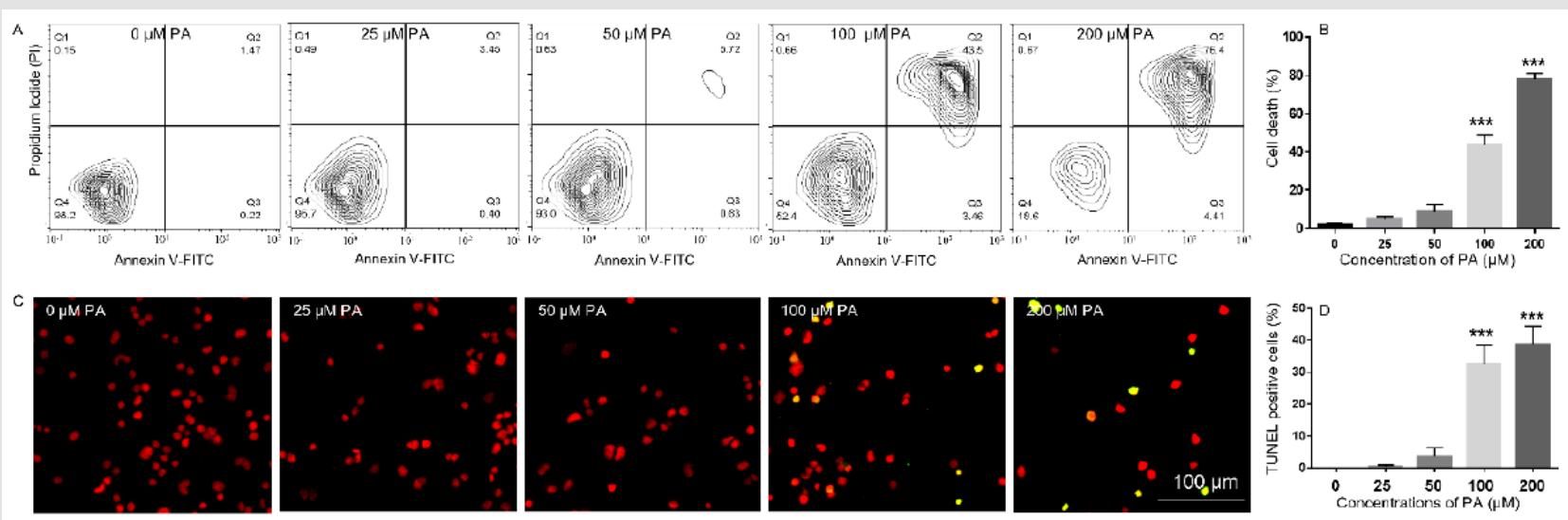

Figure 2: Analysis of PA-induced N2a cell death by Annexin V/propidium iodide (PI) staining (A-B) and TUNEL assay (C-D). (A-B), N2a cells were treated with $0,25,50,100$, and $200 \mu \mathrm{M}$ PA for $24 \mathrm{~h}$ in serum-free media followed by staining with FITCconjugated Annexin V and Propidium Iodide (PI) and analysis of fluorescence by flow cytometry with representative contour plots of compensated FITC vs. PI channels shown in A and combined percentage of FITC ${ }^{+} / \mathrm{PI}^{-}$and FITC $/ \mathrm{PI}^{+}$cells shown in B. (C-D), N2a cells were treated with 0, 25, 50, 100, and $200 \mu \mathrm{M}$ PA for $24 \mathrm{~h}$ in serum-free media followed by TUNEL assay with representative confocal microscopy images of Fluorescein-12-dUTP and PI staining shown in C, and percentage of TUNEL ${ }^{+}$ cells shown in $\mathrm{D}$. The values presented were representative of three independent experiments (mean \pm SEM). Statistical analysis was performed using one-way ANOVA followed by Tukey's multiple comparison test. ${ }^{* * *} \mathrm{p}<0.001$ vs. control.

\section{PA-Induced Decrease of N2a Viability Was Not Alleviated by NAC or 4-PBA}
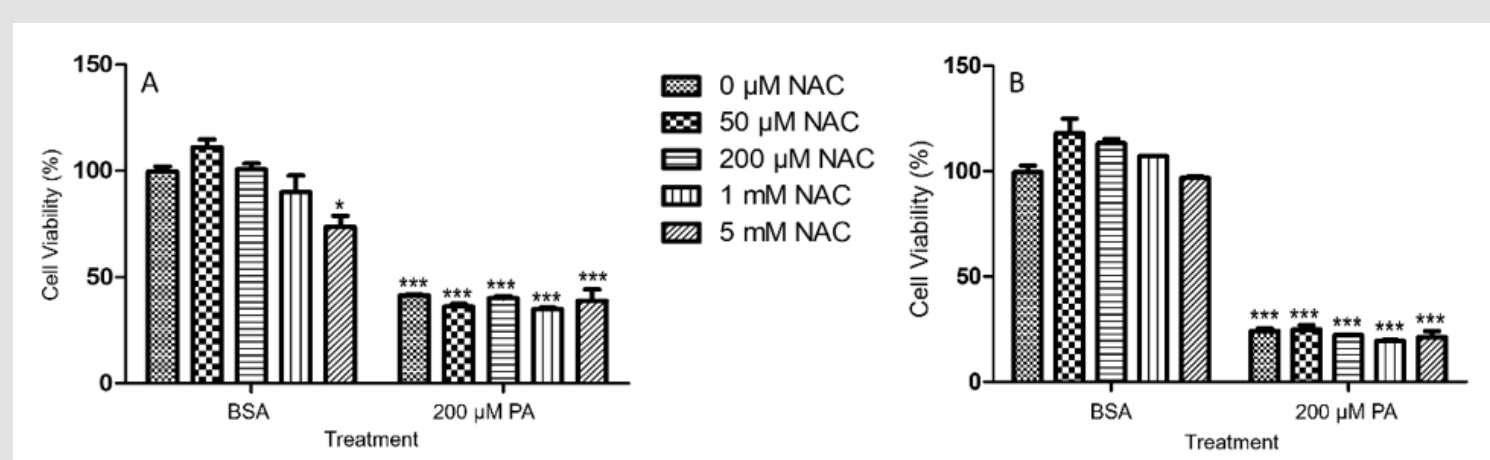

Figure 3: PA-induced decrease of viability in N2a cells was not attenuated by NAC (A) or 4-PBA (B). N2a cells were pre-treated with different concentrations of NAC (A) or 4-PBA (B) for $0.5 \mathrm{~h}$ followed by treatment with $200 \mu \mathrm{M}$ PA or BSA as control for $24 \mathrm{~h}$ in serum free media, and their viability was assessed by MTT assay. The values presented were representative of three independent experiments with triplicate measurements (mean \pm SEM). Statistical analysis was performed using two-way ANOVA followed by Tukey's multiple comparison test. ${ }^{* * *} \mathrm{p}<0.001 \mathrm{vs}$. control. 
To determine whether PA-induced decrease of N2a viability could be alleviated by antioxidants, N2a cells were pre-treated with $50 \mu \mathrm{M}, 200 \mu \mathrm{M}, 1 \mathrm{mM}$, or $5 \mathrm{mM}$ of N-acetyl cysteine (NAC) for $0.5 \mathrm{~h}$ followed by treatment with $200 \mu \mathrm{M}$ PA or BSA for $24 \mathrm{~h}$ in serum free media. NAC alone slightly decreased N2a viability at 5 $\mathrm{mM}$ as compared to the BSA control without any NAC. NAC at all concentrations tested did not significantly attenuate PA-induced decrease in N2a viability (Figure 3A). To determine whether PAinduced decrease of $\mathrm{N} 2 \mathrm{a}$ viability could be alleviated by inhibitors of endoplasmic reticulum (ER) stress, N2a cells were pre-treated with $10 \mu \mathrm{M}, 50 \mu \mathrm{M}, 200 \mu \mathrm{M}$, or $1 \mathrm{mM}$ 4-phenylbutyric acid (4-PBA) for $0.5 \mathrm{~h}$ followed by treatment with $200 \mu \mathrm{M}$ PA or BSA for $24 \mathrm{~h}$ in serum free media. While PA induced significant decrease of $\mathrm{N} 2 \mathrm{a}$ viability, 4-PBA at all concentrations tested did not significantly attenuate PA-induced decrease in N2a viability (Figure 3B).

\section{Unsaturated Fatty Acids Abrogated PA-Induced Decrease of N2a Viability}

To investigate whether unsaturated fatty acids (UFA) mitigated PA-induced decrease of N2a viability, N2a cells were treated with $25,50,100$, or $200 \mu \mathrm{M}$ of different UFA, such as LA, OA, ALA, and DHA, together with $200 \mu \mathrm{M}$ PA or BSA in serum free media for $24 \mathrm{~h}$, and their viability was determined by MTT assay. Each UFA examined was found to significantly attenuate PA-induced decrease of $\mathrm{N} 2 \mathrm{a}$ viability starting at concentrations as low as $25 \mu \mathrm{M}$. At 100 and $200 \mu \mathrm{M}, \mathrm{OA}, \mathrm{LA}, \mathrm{ALA}$, and DHA abolished the PA-induced decrease in N2a viability (Figure 4).
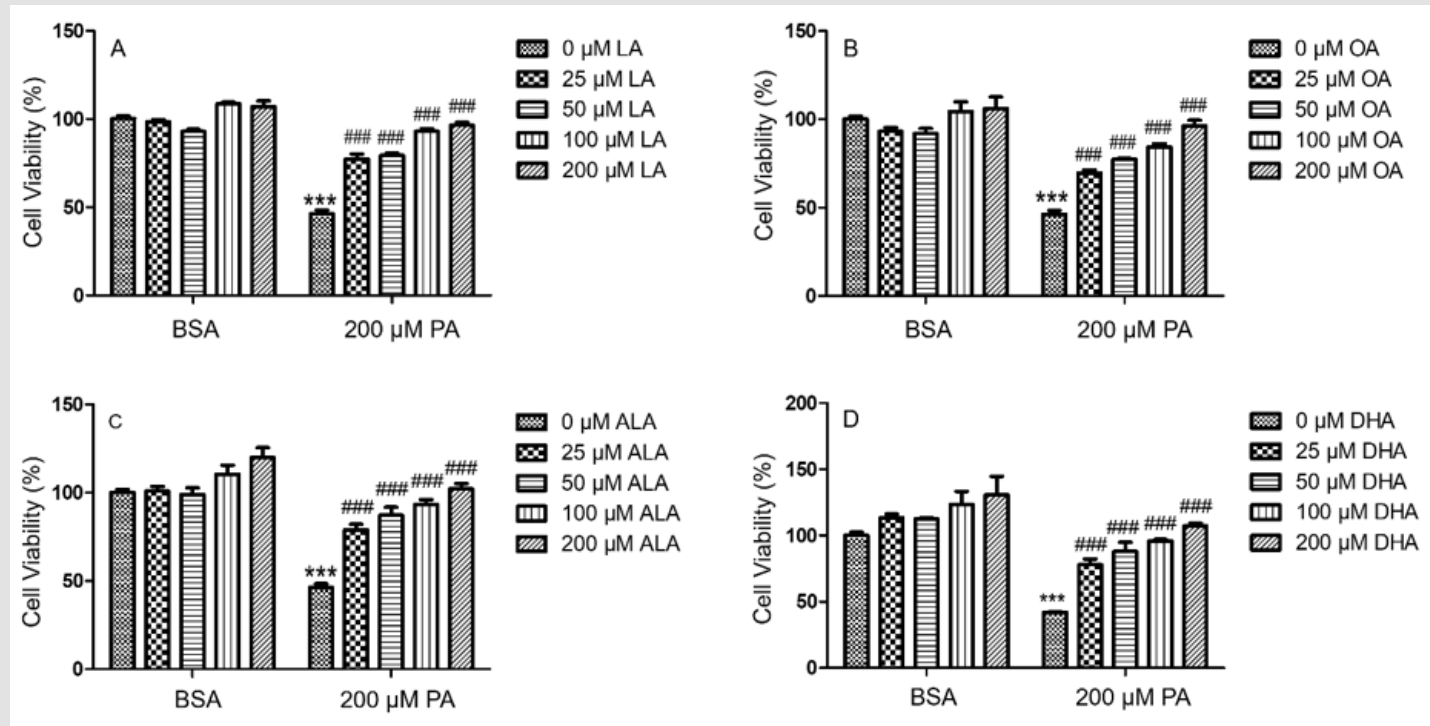

Figure 4: PA-induced decrease in BV2 viability was abolished by LA (A), OA (B), ALA (C), and DHA (D). BV2 cells were treated with $0,25,50,100$, or $200 \mu \mathrm{M}$ of LA (A), OA (B), ALA (C) and DHA (D) together with $200 \mu \mathrm{M}$ PA or equivalent volume of BSA control in serum-free media for $24 \mathrm{~h}$, and their viability was assessed by MTT assay. The values presented were representative of three independent experiments (mean \pm SEM). Statistical analysis was performed using two-way ANOVA followed by Tukey's multiple comparison test. ${ }^{* * *} p<0.001$ vs. BSA control; \#\#\#p <0.001 vs. $200 \mu$ M PA.

\section{SFA and UFA Differentially Modulated Neutral Lipid Accumulation}

To examine the effects of different FFA on the accumulation of neutral lipids, N2A cells were treated with $200 \mu \mathrm{M}$ FFA or BSA control for $6 \mathrm{~h}$, then incubated with different lipophilic dyes, including 4,4-Difluoro-1,3,5,7,8-Pentamethyl-4-Bora-3a,4a-Diazas-Indacene (BODIPYTM 493/503), Oil Red O, and Nile Red, and examined under microscopy. BSA-treated cells exhibited only basal level neutral lipid staining by BODIPY ${ }^{\mathrm{TM}} 493 / 503$, Oil Red O, and Nile Red. Neutral lipid staining in PA-treated cells was comparable to basal level staining in BSA controls as detected by BODIPY ${ }^{\mathrm{TM}}$ 493/503, Oil Red O, and Nile Red. In contrast to BSA and PA, cells treated with LA, OA, ALA, or DHA all exhibited more intense neutral lipid staining as detected by all three lipophilic dyes. Moreover, neutral lipid staining appeared to be concentrated in particulates in UFA-treated cells and more diffuse in PA-treated cells, suggesting that UFA not only increased the amount of neutral lipids, but also promoted formation of LD (Figure 5A).

Differential neutral lipid accumulation following UFA and SFA treatment was also quantitated by BODIPY ${ }^{\mathrm{TM}} 493 / 503$ staining via flow cytometry. PA did not dramatically alter the fluorescence intensity of BODIPY ${ }^{\mathrm{TM}} 493 / 503$ staining at all concentrations tested (Figures 5B \& 5C). Consistent with microscopic examination of neutral lipid staining as shown in Figure 5A, the amount of BODIPY $^{\mathrm{TM}} 493 / 503$ staining was significantly enhanced by LA, $\mathrm{OA}$, and DHA at $200 \mu \mathrm{M}$ as compared to BSA control (Figures 5B 
\& 5C). BODIPY ${ }^{\mathrm{TM}}$ 493/503 staining appeared consistently greater in ALA-treated cells compared to BSA control, but the amplitude of this increase did not reach statistical significance (Figures $5 \mathrm{~B}$
\& 5C). These data suggest that UFA, but not SFA, promoted the accumulation of neutral lipids in N2a cells.

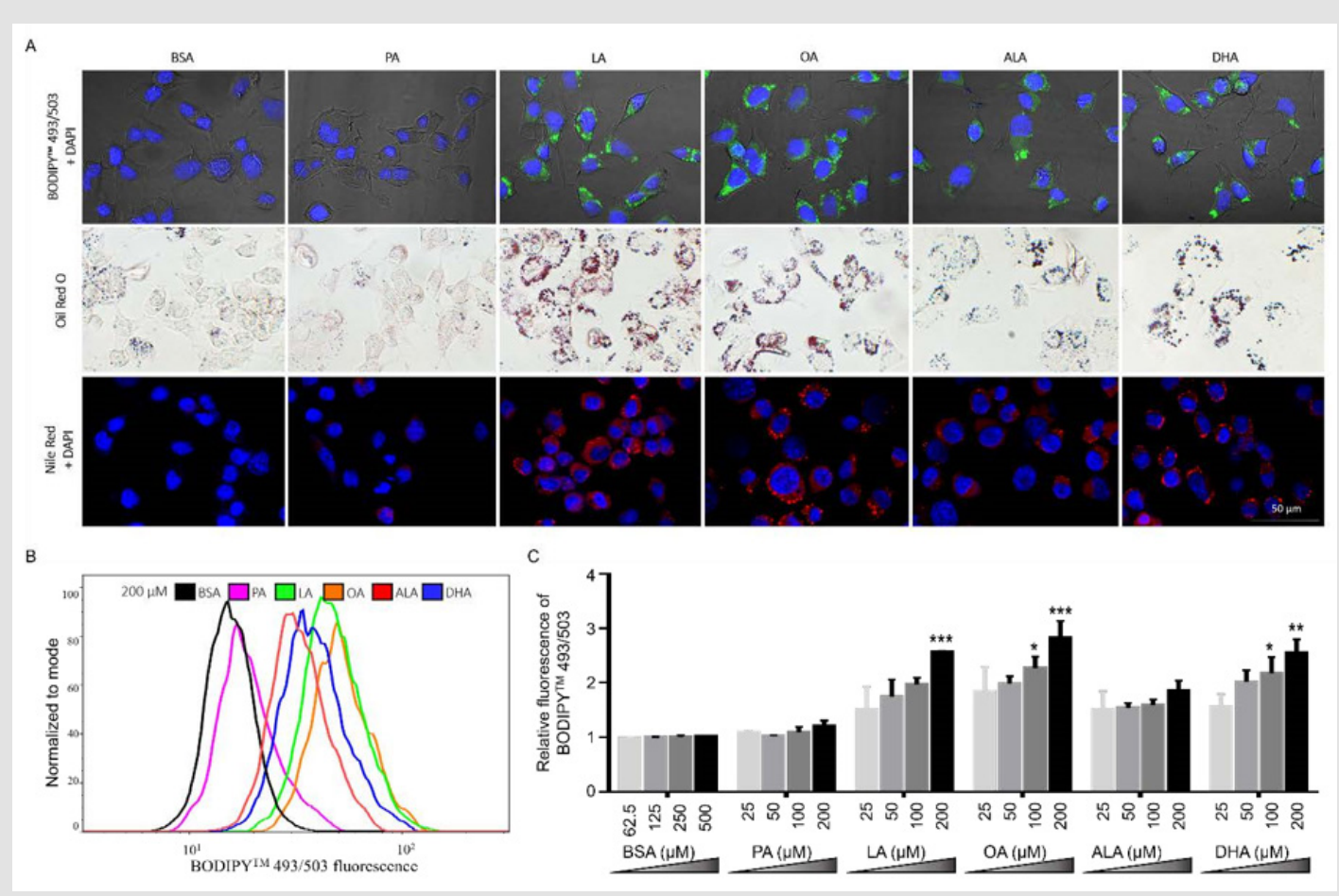

Figure 5: Neutral lipid accumulation in N2a cells following treatment with different FFA.

(A) Representative images of N2A cells treated with $200 \mu \mathrm{M}$ PA, LA, OA, ALA, DHA, or BSA control for 6 h, stained with DAPI (blue) and BODIPYTM 493/503 (green) and visualized under confocal microscopy, or stained with Oil Red O (red) and visualized under bright-field microscopy, or stained with DAPI (blue) and Nile Red (red) and visualized under confocal microscopy.

(B) Representative overlayed flow cytometry histograms of cells treated with $200 \mu \mathrm{M}$ FFA or BSA for $6 \mathrm{~h}$ and stained with BODIPYTM 493/503.

(C) Relative quantitation of lipid accumulation in N2a cells treated with different concentrations of FFA or BSA control for $6 \mathrm{~h}$ as analyzed by flow cytometry following BODIPYTM 493/503 staining. Presented data (mean \pm SEM) were representative of three independent experiments. Statistical analysis was performed using two-way ANOVA followed by Tukey's multiple comparison test. ${ }^{*} \mathrm{p}<0.05 \mathrm{vs}$. corresponding BSA control; ${ }^{* *} \mathrm{p}<0.01 \mathrm{vs}$. corresponding BSA control; ${ }^{* * *} \mathrm{p}<0.001 \mathrm{vs}$. corresponding BSA control.

\section{UFA did not Significantly Increase the Amount of Neutral Lipid Accumulation in PA-Treated Cells}

To examine whether UFA-induced synthesis of neutral lipid accumulation was involved in the protective effects of UFA against PA-induced decrease of N2a viability, N2a cells were treated with $200 \mu \mathrm{M}$ LA, OA, ALA, or DHA with or without $200 \mu \mathrm{M}$ PA for $24 \mathrm{~h}$, stained with Nile Red and DAPI, and then visualized under confocal microscopy. While UFA enhanced neutral lipid accumulation and LD formation compared to cells treated with BSA or PA alone, the total amount of neutral lipid staining in cells co-treated with UFA and PA appeared comparable to that in PA-treated cells, suggesting that UFA may not enhance neutral lipid accumulation in PA-treated cells. The neutral lipid staining did appear to be more concentrated in particulates in cells co-treated with UFA and PA but more diffuse in PA-treated cells, suggesting that UFA induced LD formation in PA-treated cells (Figure 6A). Neutral lipid accumulation was also quantified by flow cytometry following BODIPY ${ }^{\mathrm{TM}} 493 / 503$ staining, which confirmed that the amount of neutral lipid staining in cells co-treated with UFA and PA was comparable to that in PAtreated cells (Figures 6B \& 6C). These studies suggest that UFA may induce LD formation in PA-treated cells even though UFA did not seem to enhance the total amount of neutral lipid staining in these cells in PA-treated cells. 


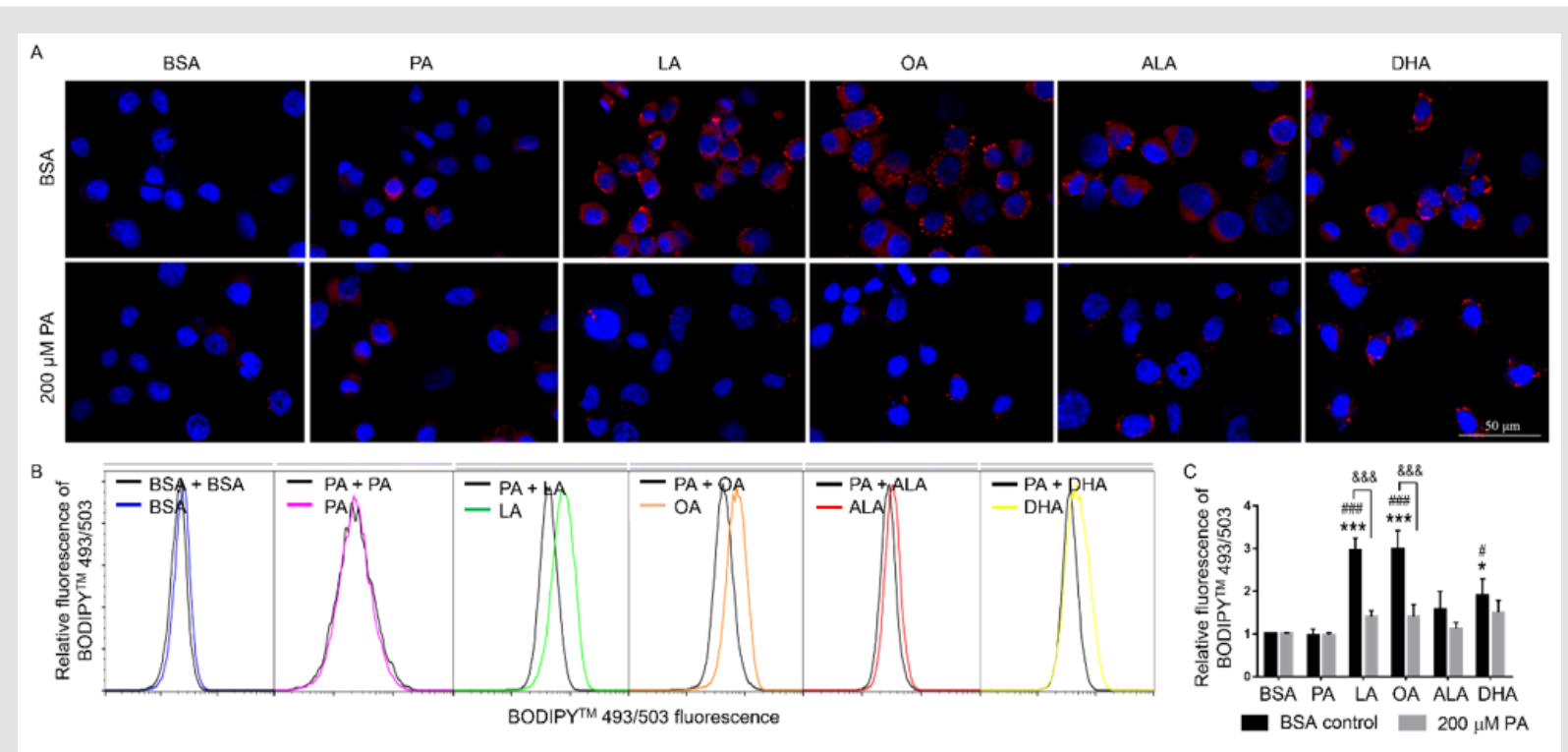

Figure 6: Neutral lipid accumulation in N2A cells co-treated with $200 \mu \mathrm{M}$ PA and different FFA.

A. Representative images of N2A cells co-treated with different FFA together with $200 \mu \mathrm{M}$ PA or BSA for $6 \mathrm{~h}$, stained with Nile red (red) and DAPI (blue), and visualized under confocal microscopy.

B. Representative flow cytometry histograms of cells co-treated with different FFA together with $200 \mu \mathrm{M}$ PA or BSA as control for $6 \mathrm{~h}$ and stained with BODIPYTM 493/503.

C. Relative quantitation of lipid accumulation as analyzed by flow cytometry following BODIPYTM 493/503 staining. Presented data (mean \pm SEM) were representative of three independent experiments. Statistical analysis was performed using two-way ANOVA followed by Tukey's multiple comparison test. ${ }^{*} p<0.05$ vs. BSA control; ${ }^{* * *} p<0.001$ vs. BSA control; \# $p<$ 0.05 vs. $200 \mu$ M PA; \#\#\#p < 0.001 vs. $200 \mu$ M PA; \&\&\& $p<0.001$ vs. corresponding BSA-treated cells.

\section{Discussion}

In the brain, lipids are involved in many functions including its development, neurogenesis, synaptogenesis, myelin sheath formation, and signal transduction [24]. Alterations of lipid homeostasis are associated with brain injury and with metabolic and neurologic disorders [25]. The goal of this study was to examine how UFA and SFA differentially affected the viability and neutral lipid accumulation in N2a cells. Our studies showed that PA, a SFA, significantly decreased N2a cell viability and induced significant cell death while the UFA, such as LA, OA, ALA, and DHA, rescued PA-induced decrease of $\mathrm{N} 2 \mathrm{a}$ viability. We also found that UFA significantly increased neutral lipid accumulation and LD formation while PA did not. Moreover, the amount of neutral lipid staining in cells co-treated with UFA and PA was comparable to that in BSA- and PA-treated cells with the staining more in particulates in cells co-treated with UFA and PA, suggesting LD formation.

PA has been shown to induce ER stress in pancreatic $\beta$ cells [26] and osteoblast-like Saos-2 cells [27] as well as oxidative stress in endometrial cells [28] and hepatocytes [29]. Inhibition of oxidative stress by NAC, an antioxidant, and ER stress by 4-PBA reduces PAinduced apoptosis in $\mathrm{H} 9 \mathrm{c} 2$ cardiomyoblast cells [30]. However, neither NAC nor 4-PBA had any significant impact on PA-induced decrease of viability in N2a cells, suggesting that ER stress and oxidative stress may not play a key role in PA-induced lipotoxicity in N2a cells. There have been studies reporting that UFA-promoted sequestration of excess PA into neutral lipids and LD may represent a mechanism by which UFA protects against PA lipotoxicity [15-18]. In this study, we showed that LA, OA, and DHA, but not PA, significantly increased neutral lipid accumulation and LD formation. However, the amount of neutral lipid staining in cells cotreated with UFA and PA was not significantly different from that in PA-treated cells, suggesting that UFA may not significantly enhance the total amount of neutral lipid accumulation in PA-treated cells.

Much of the neutral lipid staining in cells co-treated with UFA and PA did appear more concentrated in particulates than PAtreated cells, suggesting that UFA induced LD formation in PAtreated cells. Future studies may further explore whether UFAinduced LD formation may contribute to UFA-mediated protection against PA lipotoxicity. An alternative mechanism may involve ceramide formation in PA lipotoxicity. PA has been reported to increase ceramide content in murine enteroendocrine cells, which is reduced by OA [31]. Both cell culture and animal studies suggest that DHA may attenuate PA- or high fat diet-induced ceramide lipotoxicity [32]. 
In summary, our study demonstrated that PA decreased cell viability and induced cell death in N2a cells. PA-induced lipotoxicity was not alleviated by pre-treatment with NAC, an antioxidant, or 4-PBA, an inhibitor of ER stress, suggesting that oxidative stress and ER stress may not play a key role in PA lipotoxicity in N2a cells. In contrast, all the UFAs tested, namely LA, OA, ALA, and DHA, protected N2a cells against PA lipotoxicity. We also found that LA, OA, and DHA, but not PA, significantly enhanced neutral lipid accumulation and LD formation, that UFA did not enhance the total amount of neutral lipid accumulation in PA-treated cells, and that UFA may induce LD formation in PA-treated cells.

\section{Acknowledgment}

This study was supported by the research fund from Department of Biological Sciences at Seton Hall University.

\section{Author Contributions}

H. Z. conceived the study. H.Z. and C.U. designed the experiments. C.U. performed the experiments. C.U. and H.Z. analyzed the data and drafted the manuscript. All authors provided input into and approved the final draft of the manuscript.

\section{Disclosure of Conflict of Interest}

The authors have stated explicitly that there are no conflicts of interest in connection with this article.

\section{References}

1. Wolters, M, K Vyncke, G Eiben, L Iacoviello, W Ahrens, et al. (2014) Reference values of whole-blood fatty acids by age and sex from European children aged 3-8 years. Int J Obes (Lond) 38: S86-S98.

2. Guo Y (2009) Lipid droplets at a glance. J Cell Sci 122(Pt 6): 749-752.

3. Brookheart RT, CI Michel, JE Schaffer (2009) As a matter of fat. Cell Metab 10(1): 9-12.

4. Engin AB (2017) What Is Lipotoxicity? Adv Exp Med Biol 960: 197-220.

5. Lieben Louis X (2019) Resveratrol prevents palmitic-acid-induced cardiomyocyte contractile impairment. Can J Physiol Pharmacol 97(12): 1132-1140.

6. Malhi H, GJ Gores (2008) Molecular mechanisms of lipotoxicity in nonalcoholic fatty liver disease. Semin Liver Dis 28(4): 360-369.

7. Wang Z, Xiuying Xian, Zhaoxia Du, Xueer Wang, Aijun Hao, et al. (2014) Palmitic acid affects proliferation and differentiation of neural stem cells in vitro. J Neurosci Res 92(5): 574-586.

8. Yuan Q, Zhaoxia Du, Eng-Ang Ling, Qian Liu, Aijun Hao, et al. (2013) Palmitic acid increases apoptosis of neural stem cells via activating c-Jun N-terminal kinase. Stem Cell Res 10(2): 257-266.

9. Crupi R, A Marino, S Cuzzocrea (2013) n-3 fatty acids: role in neurogenesis and neuroplasticity. Curr Med Chem 20(24): 2953-2963.

10. Ben Dror K, R Birk (2019) Oleic acid ameliorates palmitic acid-induced ER stress and inflammation markers in naive and cerulein-treated exocrine pancreas cells. Biosci Rep 39(5): BSR20190054.

11. Zeng X, Lan Li, Jingping Liu, Yanrong Lu, Jingqiu, et al. (2020) Oleic acid ameliorates palmitic acid induced hepatocellular lipotoxicity by inhibition of ER stress and pyroptosis. Nutr Metab (Lond) 17: 11.
12. Hunt WT, Amit Kamboj, Hope D Anderson, Christopher M Anderson (2010) Protection of cortical neurons from excitotoxicity by conjugated linoleic acid. J Neurochem 115(1): 123-130.

13. Jarc E, T Petan (2019) Lipid Droplets and the Management of Cellular Stress. Yale J Biol Med 92(3): 435-452.

14. Olzmann JA, P Carvalho (2019) Dynamics and functions of lipid droplets. Nat Rev Mol Cell Biol 20(3): 137-155.

15. Cheon HG, YS Cho (2014) Protection of palmitic acid-mediated lipotoxicity by arachidonic acid via channeling of palmitic acid into triglycerides in C2C12. J Biomed Sci 21: 13.

16. Colvin BN (2017) Oleate attenuates palmitate-induced endoplasmic reticulum stress and apoptosis in placental trophoblasts. Reproduction 153(4): 369-380.

17. Listenberger LL, Sarah E Lewis, Sylvaine Cases, Robert V Farese Jr, Daniel S Ory, et al. (2003) Triglyceride accumulation protects against fatty acidinduced lipotoxicity. Proc Natl Acad Sci U S A 100(6): 3077-3082.

18. Nemecz M, Nicoleta Alexandru, Alexandru Filippi, Gabriela Tanko, Adriana Georgescu, et al. (2018) The Distinct Effects of Palmitic and Oleic Acid on Pancreatic Beta Cell Function: The Elucidation of Associated Mechanisms and Effector Molecules. Front Pharmacol 9: 1554.

19. Karmi A, Pirjo Nuutila, Jussi Hirvonen, Barbara A Fielding, Kirsi Virtanen, et al. (2010) Increased brain fatty acid uptake in metabolic syndrome. Diabetes 59(9): 2171-2177.

20. Farmer BC (2020) Lipid Droplets in Neurodegenerative Disorders. Front Neurosci 14: 742.

21. Beraldi EJ (2020) Colonic neuronal loss and delayed motility induced by high-fat diet occur independently of changes in the major groups of microbiota in Swiss mice. Neurogastroenterol Motil 32(2): e13745.

22. McLean FH, Rosamund F Langston, Domenico Sergi, Cibell Resch, Christine, et al. (2019) A high-fat diet induces rapid changes in the mouse hypothalamic proteome. Nutr Metab (Lond) 16: 26.

23. Choi SJ (2010) Cultured hypothalamic neurons are resistant to inflammation and insulin resistance induced by saturated fatty acids. Am J Physiol Endocrinol Metab 298(6): E1122-E1130.

24. Hussain G, Tao Sun, Atif Muhammad, Azhar Rasul, Ali Imran, et al. (2020) Lipids as biomarkers of brain disorders. Crit Rev Food Sci Nutr 60(3): 351374 .

25. Adibhatla, RM JF Hatcher (2008) Phospholipase A (2), reactive oxygen species, and lipid peroxidation in CNS pathologies. BMB Rep 41(8): 560567.

26. Suzuki E (2018) Docosahexaenoic Acid Reduces Palmitic Acid-Induced Endoplasmic Reticulum Stress in Pancreatic Beta Cells. Kobe J Med Sci 64(2): E43-E55.

27. Yang L, Xiuwen Zhan, Zhenzhen Jiang, Xiang Gu, Shengyuan Liu, et al. (2018) Palmitic acid induces human osteoblast-like Saos- 2 cell apoptosis via endoplasmic reticulum stress and autophagy. Cell Stress Chaperones 23(6): 1283-1294.

28. Li P, Yang Guo, Miao Long, Shuhua Yang, Jianbin He, et al. (2019) Palmitic Acid and beta-Hydroxybutyrate Induce Inflammatory Responses in Bovine Endometrial Cells by Activating Oxidative Stress-Mediated NFkappaB Signaling. Molecules 24(13): 2421.

29. Chen X, Jingping Liu, Jingqiu Cheng, Yanrong Lu, Younan Chen, et al. (2018) Oleic acid protects saturated fatty acid mediated lipotoxicity in hepatocytes and rat of non-alcoholic steatohepatitis. Life Sci 203: 291304.

30. Yang L (2019) Oxidative and endoplasmic reticulum stresses are involved in palmitic acid-induced H9c2 cell apoptosis. Biosci Rep 39(5): BSR20190225. 
31. Thombare K (2017) Long chain saturated, and unsaturated fatty acids exert opposing effects on viability and function of GLP-1-producing cells: Mechanisms of lipotoxicity. PLoS One 12(5): e0177605.

ISSN: 2574-1241

DOI: 10.26717/BJSTR.2021.37.006017

Heping Zhou. Biomed J Sci \& Tech Res

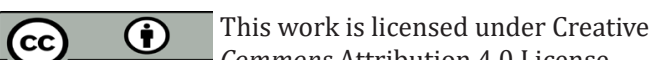

Submission Link: https://biomedres.us/submit-manuscript.php
32. Walchuk C, Y Wang, M Suh (2021) The impact of EPA and DHA on ceramide lipotoxicity in the metabolic syndrome. Br J Nutr 125(8): 863875 .

$\begin{array}{ll}\text { BIOMEDICAL } & \text { Assets of Publishing with us } \\ \text { RESEARCHES } & \text { - Global archiving of articles } \\ \text { - Immediate, unrestricted online access }\end{array}$

